\title{
Valores normativos del test de Toulouse-Piéron de atención selectiva y sostenida en una muestra de estudiantes de educación física de Santiago de Chile
}

\author{
Normative values of Toulouse-Piéron's test of attention selective and supported in a \\ students' sample of physical education of Santiago of Chile \\ * Fernando Maureira Cid, *Ashley Bobadilla Manríquez, \\ * María Angélica Ramírez Nahuelñir, * Diego Fuentealba Colil
}

Maurerira, F., Bobadilla, A., Ramírez, MA., Fuentealba, D. (2019). Valores normativos del test de Toulouse-Piéron de atención selectiva y sostenida en una muestra de estudiantes de educación física de Santiago de Chile. Revista Ciencias de la Actividad Física UCM, N²0(1) enero-junio, 1-7. DOI: http://doi.org/10.29035/rcaf.20.1.2

\section{RESUMEN}

El objetivo del presente estudio fue determinar valores normativos para cada puntaje del test de atención de Toulouse-Piéron para estudiantes de educación física de una Universidad de Santiago de Chile. La muestra estuvo constituida por 125 estudiantes, de los cuales el $68 \%$ corresponden a varones y un $32 \%$ a damas. Se utilizó el test de atención de Tolulouse-Piéron, utilizando los 2 primeros minutos para evaluar la atención selectiva y su ejecución total (10 minutos) para evaluar la atención sostenida. Los resultados muestran una media del Índice Global de Atención y

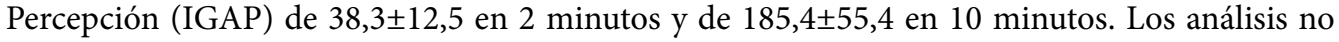
muestran diferencias por sexo o edad en ninguna de las 2 atenciones evaluadas. La atención selectiva presenta un IGAP de 31,5 para el percentil 25, de 39 para el percentil 50 y de 46 para el percentil 75. Por su parte, la atención sostenida presenta un IGAP de 158 para el percentil 25, de 190 para el percentil 50 y de 217 para el percentil 75. Son necesarias más investigaciones con muestras de diversas escuelas de educación física del país.

\section{PALABRAS CLAVE}

Atención selectiva, atención sostenida, educación física, baremos.

\begin{abstract}
The aim of the present study was to determine normative values for every puntaje of the test of Toulouse-Piéron's attention for students of physical education of a University of Santiago of Chile. The sample was constituted by 125 students, of which $68 \%$ corresponds to males and 32 $\%$ to ladies. There was in use the test of Tolulouse-Piéron's attention, using the first two minutes to evaluate the selective attention and his total execution (ten minutes) to evaluate the supported attention. The results show an average of the Global Index of Attention and Perception (GIAP) of $38,3 \pm 12,5$ in two minutes and of $185,4 \pm 55,4$ in ten minutes. The analyses do not show differences for sex or age in any of two evaluated attentions. The selective attention presents an GIAP of 31,5 for the percentile 25, of 39 for the percentile 50 and of 46 for the percentile 75 . For your part, the supported attention presents an GIAP of 158 for the percentile 25, of 190 for the percentile 50 and of 217 for the percentile 75 . More investigations are necessary with samples of diverse schools of physical education of the country.
\end{abstract}

\section{Key words}

Selective attention, supported attention, physical education, scales. 
Maurerira, F., Bobadilla, A., Ramírez, MA., Fuentealba, D. (2019). Valores normativos del test de Toulouse-Piéron de atención selectiva y sostenida en una muestra de estudiantes de educación física de Santiago de Chile. Revista Ciencias de la Actividad Física UCM, N ${ }^{\circ}$ 20(1) enero-junio, 1-7. ISSN: 0719-4013. DOI: http://doi.org/10.29035/rcaf.20.1.2

\section{INTRODUCCIÓN}

La atención puede ser definida como la focalización selectiva de nuestras percepciones, seleccionando alguna información particular del entorno, desechando las restantes (Estévez, García \& Junque, 1997, citado en Maureira \& Flores, 2017). De esta forma, nuestro cerebro escoge ciertos estímulos, siendo estos los más importantes para dirigir la conducta en ese momento (Maureira, Flores \& Trujillo, 2014). Cuando niños somos capaces de fijar la atención por cortos períodos de tiempo, pero a medida que maduramos esta situación se vuelve más controlable, mejorando la capacidad de fijar y mantener la atención en un objeto seleccionado (Fuenmayor \& Villasmil, 2008).

Actualmente se acepta que la atención no es una función unitaria y que puede clasificarse en 2 grandes grupos: atención involuntaria y atención voluntaria. La primera corresponde al estado de alerta que ocurre desde que despertamos en la mañana y nos predispone para recibir los estímulos (Maureira \& Flores, 2016); la atención voluntaria se clasifica en diversos tipos: a) atención focal, que ocurre cuando un sujeto presta atención a un solo estímulo, desechando otros (Maureira, 2018). También es llamada concentración (Ardila \& Ostrosky, 2012); b) atención selectiva o capacidad de buscar un estímulo ignorando los demás; c) atención sostenida, definida como la capacidad de ejecutar una conducta cognitiva a lo largo de diversas ventanas temporales en contra de la fatiga (Maureira \& Flores, 2016); d) atención alternante o capacidad de cambiar el foco atencional de un estímulo a otro rápidamente $\mathrm{y} ; \mathrm{e}$ ) atención dividida, que corresponde a la capacidad de focalizar dos o más estímulos al mismo tiempo (Ardila \& Ostrosky, 2012).

Actualmente en Chile casi no existen estudios sobre la atención en estudiantes universitarios, ya que en forma general se suele estudiar a los niños/as (debido a su proceso de desarrollo y relación de la atención con el rendimiento escolar) y adultos mayores (por su deterioro cognitivo, donde se ve afectada la atención). Sin embargo, en la edad universitaria, aún es importante la relación atención- rendimiento académico y, en estudiantes de educación física, esto se hace más particular, debido a las características creativas, improvisadoras, participativas, animadoras, prácticas, realistas, decididas y concretas que poseen estos jóvenes (Maureira, Flores, González, Palma \& Fernández, 2018).

La revisión de la literatura mostró algunos trabajos de la atención evaluados con el test de Toulouse-Piéron. Teixeira, Gobbi, Pereira, Vital, Hernandéz, Shigematsu, et al. (2017) evaluaron a 41 adultos mayores entre 60 y 89 años, los cuales fueron agrupados en 2 conjuntos: el grupo 1 mostró una media de $63,0 \pm 32,1$ respuestas correctas en la primera medición y $76,4 \pm 4,5$ respuestas correctas en la segunda medición; el grupo 2 obtuvo 57,4 $\pm 21,3$ respuestas correctas en la primera medición y $54,5 \pm 25,9$ en la segunda medición.

Montoya, Araya \& Salazar (2007) evaluaron a 57 damas y varones adultos (30 realizaban danza aeróbica y 27 , yoga) describiendo una media de 81,4 puntos en el test de ToulousePiéron, en una primera medición del grupo de danza y 88,2 en una segunda evaluación. Por su parte, el grupo de yoga obtuvo 82,1 puntos en su primera medición y 86,7 en su segunda evaluación. Un estudio de Hernández, Castillo \& Mendoza (2017), en que evaluaron a 58 médicos residentes e internos de pregrado, separándolos en los que habían dormido menos de 2 horas a causa de sus guardias médicas, y los que habían dormido más de ese tiempo. Los resultados del test de atención de ToulousePiéron mostraron valores medios de $35,5 \pm 14,5$ en el grupo con disminución de tiempo de sueño y de 43,5 $\pm 9,86$ en el grupo control.

Rodríguez \& Montoya (2006) aplicaron el test de Toulouse-Piéron, el test de percepción de diferencias de caras y el test de Stroop a 36 deportistas con $21,9 \pm 4,01$ años. El puntaje del test de Toulouse-Piéron se obtuvo con el número total de aciertos, menos el número de errores, dividido por el número de aciertos. La muestra fue dividida en 3 grupos, con una media de $0,94 \pm 0,06$ en la primera medición del grupo 1 y $0,97 \pm 0,03$ en la segunda medi- 
ción; $0,96 \pm 0,04$ en la primera medición del grupo 2 y $0,97 \pm 0,03$ en la segunda evaluación; $0,94 \pm 0,06$ en la primera medición del grupo 3 y $0,93 \pm 0,06$ en la segunda evaluación. Un estudio de Maureira, Flores \& Trujillo (2014) aplicó el test de atención de Toulouse-Piéron a 151 estudiantes de educación física de la Universidad SEK en Chile, mostrando 102 respuestas correctas como mínimo, 294 como máximo, con una media de $207,6 \pm 44,8$, con un percentil 5 de 129 respuestas correctas, un percentil 25 de 173 , un percentil 50 de 208, un percentil 75 de 243 y un percentil de 95 de 280.

En base a los antecedentes mencionados surge el objetivo de la presente investigación: determinar valores normativos para cada puntaje del test de atención de Toulouse-Piéron para estudiantes de educación física de una Universidad de Santiago de Chile. Esto, debido a la poca información que se encuentra en relación con la atención en estudiantes de esta población y la necesidad de contar con valores normativos para la aplicación de este instrumento en futuras investigaciones, fortaleciendo el estudio de funciones cognitivas con la implementación de pruebas y test neuropsicológicos de fácil aplicación en estudiantes y profesionales de la educación física.

\section{MÉTODO}

Muestra: de tipo no probabilística e intencionada. Estuvo constituida por 125 estudiantes de la carrera de educación física de una Universidad privada de Santiago de Chile. Del total, 85 casos corresponden a varones $(68 \%)$ y 40 a damas (32\%). La edad mínima fue de 17 años, la máxima de 23 , con una media de $18,6 \pm 1,15$.

Instrumento: se utilizó el test de atención de Toulouse-Piéron creada en 1904, la cual es considerada como una prueba adecuada para medir la atención selectiva y sostenida desde los 6 a los 90 años (León, 1995). El instrumento consta de 1200 figuras, las cuales son cuadraditos con una línea en algún lado o ángulo, existiendo dos modelos que el evaluado debe encontrar y marcar dentro de la matriz. La aplicación del instrumento durante 2 minutos evalúa la atención selectiva y su aplicación durante 10 minutos evalúa la atención sostenida. La prueba puede ser realizada en forma individual o colectiva.

Procedimiento: La recogida de datos se realizó durante el mes de marzo del 2018, en horario de clases, entre las 9:00 y 12:00 hrs. Se explicó a los estudiantes la forma de contestar el test, a los 2 minutos se les avisó para que marcasen una línea en el lugar que están contestando e inmediatamente continuaron hasta completar los 10 minutos. De esta forma fue posible extraer la información sobre la atención selectiva y sostenida. Cada estudiante firmó un consentimiento informado para participar en la investigación.

Análisis de datos: Se utilizó el programa SPSS 24.0 para Windows. Se aplicó estadística descriptiva como medias y desviaciones estándar para cada puntaje del test. También se calcularon los percentiles para establecer los baremos de la prueba. Se utilizó estadística inferencial como pruebas t para muestras independientes para comparar los puntajes según el sexo de la muestra y pruebas de ANOVA para comparar puntajes según las edades. El nivel de significancia se estableció en 0,05.

\section{RESULTADOS}

En la Tabla 1 se muestran los valores mínimos, máximos, medias y desviaciones estándar de cada una de las variables medidas con el test de Toulouse-Piéron. Los valores obtenidos en 2 minutos corresponden a la atención selectiva y en 10 minutos a la atención sostenida. El Índice Global de Atención y Percepción (IGAP) se obtiene con las respuestas correctas (C), las respuestas erradas (E) y omitidas (O) de la siguiente forma: $\mathrm{C}-(\mathrm{E}+\mathrm{O})$. Por su parte, el Índice de Control de Impulsividad (ICI) se obtiene de la siguiente forma: [(C - E) / R] $\mathrm{x} 100$, siendo $\mathrm{R}$ el número total de respuestas del evaluado. 
Maurerira, F., Bobadilla, A., Ramírez, MA., Fuentealba, D. (2019). Valores normativos del test de Toulouse-Piéron de atención selectiva y sostenida en una muestra de estudiantes de educación física de Santiago de Chile. Revista Ciencias de la Actividad Física UCM, N 20(1) enero-junio, 1-7. ISSN: 0719-4013.

DOI: http://doi.org/10.29035/rcaf.20.1.2

Tabla 1

Estadística descriptiva de los resultados de atención del total de la muestra.

\begin{tabular}{lcll}
\hline Datos & Mínimo & Máximo & \multicolumn{1}{c}{ Media } \\
\hline Respuestas correctas en 2 minutos & 24 & 78 & $46,4 \pm 9,05$ \\
\hline Respuestas erradas en 2 minutos & 0 & 4 & $0,29 \pm 0,67$ \\
\hline Respuestas omitidas en 2 minutos & 0 & 55 & $7,88 \pm 7,11$ \\
\hline IGAP & 8 & 78 & $38,3 \pm 12,52$ \\
\hline ICI & 81,8 & 100,0 & $98,7 \pm 3,12$ \\
\hline Respuestas correctas en 10 minutos & 124 & 299 & $222,0 \pm 43,39$ \\
\hline Respuestas erradas en 10 minutos & 0 & 8 & $1,25 \pm 1,74$ \\
\hline Respuestas omitidas en 10 minutos & 5 & 124 & $35,6 \pm 24,28$ \\
\hline IGPA & 28 & 291 & $185,4 \pm 55,38$ \\
\hline ICI & 90,0 & 100,0 & $98,8 \pm 1,82$ \\
\hline
\end{tabular}

Los análisis comparativos de respuestas correctas, erradas, omisiones IGAP e ICI para los 2 y 10 minutos del test de Toulouse-Piéron no muestran diferencias significativas según el sexo de la muestra ( $\mathrm{p}>0,05)$. Misma situación que ocurre al comparar los resultados según la edad de la muestra ( $p>0,05)$. Esto lleva a establecer baremos únicos para cada variable estudiada.

En las Tablas 2 y 3 se observan los valores normativos para la atención selectiva y sostenida evaluada con el test de Toulouse-Piéron. Esto permite clasificar los puntajes en cinco rangos:

a) superior: si su puntaje es $\geq$ al percentil 95 ;

b) medio superior: si su puntaje es $\geq$ al percentil $75 \mathrm{y}<$ al percentil 95; c) medio: si su puntaje es $>$ al percentil $25 \mathrm{y}<$ al percentil 75;

d) medio inferior: si su puntaje es $>$ al percentil $5 \mathrm{y} \leq$ al percentil 25

e) deficiente: si su puntaje es $\leq$ al percentil 5 .

En el IGAP de la atención selectiva se observa que en la categoría deficiente se encuentran 6 estudiantes $(4,8 \%)$, en la categoría medio inferior, 25 estudiantes (20\%), en la categoría medio, 72 estudiantes (57,6\%), en la categoría medio superior, 16 estudiantes $(12,8 \%)$ y en la categoría superior, 6 estudiantes $(4,8 \%)$.

Tabla 2

Baremos para cada variable del test de Toulouse-Piéron para atención selectiva (2 minutos).

\begin{tabular}{cccccc}
\hline Percentiles & Correctas & Erradas & Omitidas & IGAP & ICI \\
\hline 99 & 75,5 & 3,8 & 49,8 & 74,6 & 100,0 \\
\hline 95 & 61,0 & 2,0 & 21,2 & 54,2 & 100,0 \\
\hline 75 & 53,0 & 0,0 & 11,0 & 46,0 & 100,0 \\
\hline 50 & 47,0 & 0,0 & 6,0 & 39,0 & 100,0 \\
\hline 25 & 40,0 & 0,0 & 3,0 & 31,5 & 100,0 \\
\hline 5 & 31,8 & 0,0 & 1,0 & 16,7 & 92,8 \\
\hline
\end{tabular}


En el IGAP de la atención sostenida se observa que en la categoría deficiente se encuentran 6 estudiantes $(4,8 \%)$, en la categoría medio inferior 26 estudiantes $(20,8 \%)$, en la categoría medio 59 estudiantes (47,2\%), en la categoría medio superior 28 estudiantes $(22,4 \%)$ y en la categoría superior 6 estudiantes $(4,8 \%)$.

Tabla 3

Baremos para cada variable del test de Toulouse-Piéron para atención sostenida (10 minutos).

\begin{tabular}{cccccc}
\hline Percentiles & Correctas & Erradas & Omitidas & IGAP & ICI \\
\hline 99 & 297,7 & 8,0 & 122,8 & 288,7 & 100,0 \\
\hline 95 & 286,5 & 6,0 & 89,1 & 266,9 & 100,0 \\
\hline 75 & 253,5 & 2,0 & 45,8 & 217,0 & 100,0 \\
\hline 50 & 222,0 & 1,0 & 28,5 & 190,0 & 99,3 \\
\hline 25 & 193,0 & 0,0 & 19,0 & 158,0 & 98,1 \\
\hline 5 & 149,2 & 0,0 & 9,0 & 79,2 & 94,7 \\
\hline
\end{tabular}

\section{DISCUSIÓN}

La media de respuestas correctas en el test de atención de Toulouse-Piéron en 2 minutos fue de $46,4 \pm 9,05$ y en 10 minutos fue de $222 \pm 43,39$. Un estudio de Maureira et al. (2014) donde aplicaron el mismo instrumento durante 10 minutos, se presentaron valores de $207,6 \pm 44,84$ respuestas correctas a una muestra de estudiantes de educación física en Chile, siendo los resultados actuales ligeramente superiores. También es posible notar que la cantidad de respuestas erradas no superan las ocho durante los 10 minutos de prueba, lo que indica un buen nivel de reconocimiento visual de las figuras solicitadas. El IGAP posee una media de $38,3 \pm 12,5$ en 2 minutos y de $185,4 \pm 55,4$ en 10 minutos, y dado que existe un alto número de respuestas correctas y baja cantidad de erradas, existe un alto número de respuestas omitidas, lo que tiende a descender el puntaje del IGAP en ambos períodos de tiempo, lo cual podría indicar una excesiva velocidad al contestar el test, lo que ocasionaría el pasar por alto muchas de las figuras buscadas. Por otra parte, el ICI es muy alto (cercano al 98\% en ambos períodos de tiempo) lo que indica un bajo nivel de impulsividad, lo que se refleja en la baja cantidad de respuestas erradas.
Los estudiantes de educación física constituyen una población especial con un patrón de actividad física diferente al resto de grupos sociales, ya que dicha práctica suele ser algo habitual en ellos y numerosos estudios relacionan la práctica de ejercicio con mejores resultados en pruebas de atención (Altenburg, Chinapaw \& Singh, 2016; Bullock \& Giesbrecht, 2014; Chang, Pesce, Chiang, Kuo \& Fong, 2015; De Bruin, van del Zwan \& Bögels, 2016, etc.). Esta situación hace importante la exploración de una variable cognitiva altamente ligada al aprendizaje en estos sujetos, los cuales poseen hábitos de vida saludable diferentes a la mayoría de la población.

Las tablas normativas entregadas sirven como referencia para comparar resultados de estudios futuros o casos individuales de estudiantes de educación física. Por ejemplo, en el estudio de Maureira et al. (2014) para el período de $10 \mathrm{mi}$ nutos el percentil 50 de las respuestas correctas fue de 208, el percentil 25 fue de 173 y el 75 de 243. En el estudio actual el percentil 50 de respuestas correcta fue de 222 , el percentil 25 de 193 y el percentil 75 fue de 253 , mostrando un leve aumento en relación con la muestra de educación física de la Universidad SEK de Chile. 
Maurerira, F., Bobadilla, A., Ramírez, MA., Fuentealba, D. (2019). Valores normativos del test de Toulouse-Piéron de atención selectiva y sostenida en una muestra de estudiantes de educación física de Santiago de Chile. Revista Ciencias de la Actividad Física UCM, N ${ }^{\circ}$ 20(1) enero-junio, 1-7. ISSN: 0719-4013.

\section{CONCLUSIÓN}

Es posible concluir que la muestra actual posee índices ligeramente mayores que otro estudio de la atención sostenida realizada en estudiantes de educación física en nuestro país. No se observaron diferencias entre mujeres y hombres, ni en las diversas edades evaluadas. Las tablas de datos normativos se establecieron para cada variable evaluada con el test de atención selectiva ( 2 minutos) y sostenida (10 minutos), con un percentil 50 del IGAP fue de 39 para los 2 minutos y de 190 para los $10 \mathrm{mi}$ nutos del test de Toulouse-Piéron.

Se necesitan futuras investigaciones que abarquen diversas escuelas de educación física de nuestro país, de manera de establecer baremos nacionales que se constituyan como herramientas en futuros estudios sobre la cognición en esta población.

\section{REFERENCIAS BIBLIOGRÁFICAS}

Altenburg, T., Chinapaw, M. \& Singh, A. (2016). Effects of one versus two bouts of moderate intensity physical activity on selective attention during a school morning in Dutch primary schoolchildren: A randomized controlled trial. Journal of Science and Medicine in Sport, 19(10), 820-824.

Ardila, A. \& Ostrosky, F. (2012). Guía para el diagnóstico neuropsicológico. Miami: Florida Internacional University.

Bullock, T. \& Giesbrecht, B. (2014). Acute exercise and aerobic fitness influence selective attention during visual search. Frontiers in Psychology, 5, 1290.

Chang, Y., Pesce, C., Chiang, Y., Kuo, C. \& Fong, D. (2015). Antecedent acute cycling exercise effects attention control: an ERP study using attention network test. Frontiers in Human Neuroscience, 9, 156.
De Bruin, E., van del Zwan, J. \& Bögels, S. (2016). A RCT Comparing daily mindfulness meditations, biofeedback exercises, and daily physical exer-cise on attention control, executive functioning, mindful awareness, self-compassion, and worrying in stressed young adults. Mindfulness, 7(5), 1182-1192.

Fuenmayor, G. \& Villasmil, Y. (2008). La percepción, la atención y la memoria como procesos cognitivos utilizados para la comprensión textual. Revista de Artes y Humanidades UNICA, 9(22), 187-202.

Hernández, F., Castillo, T. \& Mendoza, K. (2017). Efecto de las guardias en habilidades cognitivas en estudiantes de especialidades médicas y médicos internos de pregrado. Revista CONAMED, 22(1), 17-22. 
León-Carrión, J. (1995). Manual de neuropsicología humana. Madrid: Siglo XXI de España Editores.

Maureira, F. (2018). Principios de neuroeducación física. Madrid: Bubok Publishing.

Maureira, F. \& Flores, E. (2016). Principios de neuropsicobiología para estudiantes de educación. Valencia: Obrapropia.

Maureira, F. \& Flores, E. (2017). Efectos del ejercicio físico sobre la atención: una revisión de los últimos años. Revista de Ciencias de la Actividad Física UCM, 18(1), 73-83.

Maureira, F., Flores, E., González, P., Palma, E. \& Fernández, M. (2018). Estilos de aprendizaje Honey-Alonso y de Ned Herrmann en estudiantes de educación física de Santiago de Chile. EmásF, Revista Digital de Educación Física, 9(52),125-133.

\section{Dirección para correspondencia}

Fernando Maureira Cid

$\mathrm{PhD}$. en Educación, especialista en

Neurociencia. Docente Escuela de Educación en Ciencias del Movimiento y Deportes,

Universidad Católica Silva Henríquez.

Santiago de Chile.

Orcid: http://orcid.org/0000-0001-7607-7416

Contacto:

maureirafernando@yahoo.es

Recibido: 12-07-2018

Aceptado: 31-01-2019
Maureira, F., Flores, E. \& Trujillo, H. (2014). Propiedades psicométricas y datos normativos del test de atención Toulouse Piéron y del test de memoria visual de Benton forma $\mathrm{D}$ en estudiantes de educación física de Chile. Gaceta de Psiquiatría Universitaria, 10(2), 238-245.

Montoya, J., Araya, G. \& Salazar, W. (2007). Efecto agudo del yoga y de la danza aeróbica sobre el estado de ánimo y el funcionamiento cognitivo en hombres y mujeres. Revista de Ciencias del Ejercicio y la Salud, 5(1), 41-46.

Rodríguez, M. \& Montoya, J. (2006). Entrenamiento en el mantenimiento de la atención en deportistas y su efectividad en el rendimiento. Acta Colombiana de Psicología 9(1), 99-112.

Teixeira, C., Gobbi, S., Pereira, J., Vital, T., Hernandéz, S., Shigematsu, R. et al. (2017). Effects of square-stepping exercise on cognitive functions of older people. Psychogeriatrics, 13, 148-156. 